\title{
Polycystic ovary syndrome: conditions, genetics and current cure
}

\begin{abstract}
Polycystic ovary syndrome (PCOS) is one of the general disorders of endocrine system affecting around 7 to $10 \%$ of reproductive age women and is the leading cause of Female infertility. ${ }^{1}$ Polycystic ovary syndrome has characteristic polycystic ovaries, hyperandrogenism and anovulation. It is a wide reproductive disorder that umbrellas many related health conditions such as obesity, type 2 diabetes, insulin resistance, cardiovascular disease, and PCOS can be related with metabolic disorders in the first-line family members. ${ }^{1,2}$ PCOS has Insulin resistance as key pathophysiology and dyslipidemia in women with PCOS can hence be found in the insulin resistant state. ${ }^{3}$ Despite the increasing cases of this syndrome and decades of research, the cause of PCOS is incompletely unknown, but various studies describe it as an internal ovarian abnormality. PCOS has no medicinal cure, ${ }^{4}$ but oral contraceptive pills (OCPs), anti-androgens and insulin controlling drug like metformin can help with improving the regularity of periods and other metabolic conditions..$^{5}$ In this review, the conditions in PCOS, genetic factors and genes involved, current treatments and future perspective of PCOS has been briefly discussed.
\end{abstract}

Volume 3 Issue 5 - 2016

\author{
Nupur Chaudhary, Imteyaz Qamar \\ School of Biotechnology, Gautam Buddha University, India \\ Correspondence: Imteyaz Qamar, School of Biotechnology, \\ Gautam Buddha University, Greater Noida, Gautam Budh Nagar, \\ UP-20I308, India, Email imteyazqamar@gmail.com
}

Received: May 21, 2016 | Published: November 25, 2016

Keywords: polycystic ovary syndrome; type 2 diabetes; hyperandrogenism; anovulation; polycystic ovaries; estrogen; insulin; hormones; cholesterol

Abbrevations: PCOS, polycystic ovary syndrome; OCPs, oral contraceptive pills; HA, hyper androgenic anovulation; SHBG, sex-hormone binding globulin; LDL, low-density lipoprotein; HDL, high-density lipoprotein; HTN, hypertension; AR, androgen receptor; $\mathrm{LH}$, luteinizing hormone; VNTR, variable number of tandem repeats; TNF-alpha, tumor necrosis factor-alpha; PPAR- $\gamma$, Peroxisome proliferator-activated receptor- $\gamma$; FST, follistatin; GCs, granulosa cells; $\mathrm{CpG}$, cytosine-phosphate-guanine; LHCGR, LH/chorio gonadotropin receptor; GO, gene ontology; TNIK, traf2- and nck-interacting kinase; $\mathrm{CAH}$, congenital adrenal hyperplasia; LOD, laparoscopic ovarian drilling

\section{Introduction}

\section{What is PCOS?}

The word 'syndrome' means set of interconnected medical symptoms or signs indicating a disease and defining polycystic ovary syndrome (PCOS) is a difficult task because of its multiform symptoms. ${ }^{1}$ It is an endocrine disorder with set of symptoms occurring due to hormonal imbalance which may further lead to a disease. There has been years of research on PCOS still complexity of pathogenesis of PCOS remain unsolved. Since PCOS is a 'syndrome', not every patient shows all the signs and symptoms of this syndrome. It has been inferred that PCOS can be a familial genetic syndrome resulted by a combination of genetic and environmental factors. ${ }^{6}$ This disorder occurs in $7-10 \%$ of women of reproductive or child bearing age and typically starts developing at puberty with signs of obesity, oligomenorrhea, hirsutism and infertility. According to the Rotterdam Criteria, women with PCOS have two, out of three, main clinical diagnostic evidence:

a) Signs of hyperandrogenism. b) Presence of polycystic ovaries in ultrasound.

c) Menstrual disorders or oligoovulation. Though according to the European Society of Endocrinology, PCOS criteria should to be revised and also, the name of the syndrome needs to be changed as it is misinforming and does not give the actual idea about this medical condition. PCOS is known with name hyper androgenic anovulation (HA). ${ }^{7}$ It is also known as Stein-Leventhal syndrome, ${ }^{8}$ in literature as in 1935, Stein and Leventhal explained the polycystic manifestation of the ovaries of women with other signs of PCOS. In this paper, various symptoms and medical conditions occurring in PCOS, published and ongoing studies of the possible genetic factors, current treatments of polycystic ovary syndrome and its future perspectives have been briefly reviewed.

\section{Symptoms}

\section{Increased levels of androgen}

Healthy ovaries produce a small amount of male sex hormones (androgens) along with the normal female sex hormone production (estrogen). In PCOS, the patient starts producing slightly more androgens. The level of androgen produced regulates the degree of PCOS in a patient. This increased androgen causes lack in ovulation, severe acne, thick and dark velvety skin in the folds, ${ }^{4}$ along with male characteristics like hirsutism (growth of extra facial and body hair) and male baldness.

\section{Menstrual complications}

Women suffering from PCOS commonly face menstrual troubles such as oligomenorrhea (fewer than nine menstrual cycles per year) or amenorrhea (no menstrual periods), ${ }^{9}$ along with other menstrual troubles like heavy or normally less bleeding, clots in menstrual discharge unbearable abdominal pains. 


\section{Infertility}

Women with PCOS have trouble in conceiving as insulin resistance and hormonal imbalance causes lack or delay in ovulation. ${ }^{10}$ This causes the patient to face uncertainty in their ovulation period and thus, difficulty in conceiving.

\section{Difficulty in weight loss}

The frequency of obesity is higher in PCOS women, and non-obese women with PCOS commonly show central adiposity and excessive body fat. The inability of insulin to function normally may cause women with PCOS tend to put on body weight or have a difficulty in losing weight.

\section{Medical conditions In PCOS \\ Obesity}

In PCOS it becomes difficult for the body to normally use the hormone insulin, a condition known as insulin resistance. Due to this, the level of androgen increases in the blood stream. Androgens are important in determination of body composition. Men have greater distribution of fat in the upper portion of their bodies (android) as compared to women where the fat is more in the lower portion of the body (gynoid). The increased level of androgen in the body causes the women with PCOS to have high chances of upper-body obesity or in the abdomen as it is where men tend to increase weight. This was observed by waist-hip ratio and increased waist circumference as compared to BMI-matched control women. ${ }^{11}$ They tend to have increased central adiposity than normal women. The inability of body to use insulin properly and normally is one reason why women with PCOS gain weight and face difficulty in losing weight. ${ }^{1}$

\section{Hyperandrogenism}

In women, signs and symptoms of hyperandrogenism normally include scalp hair loss (androgenic alopecia), acne, extra facial and body hair (hirsutism), atypically breast atrophy, high libido and others. Figure 1 shows the increased growth of facial as well as body hair as degree of PCOS related hirsutism increased (in increasing order from column 1 to column 4). High levels of androgen can be due to an increased production by both ovaries and androgens. The ovaries produce about $60 \%$ of androgens, while the adrenals contribute the remaining $40 \% .{ }^{9}$ Low presence of sex-hormone binding globulin (SHBG) can reduce testosterone binding and cause high free testosterone levels in PCOS patient.

\section{Insulin Resistance}

Insulin resistance is a condition in which the body produces insulin but the cells are not able to respond to the actions of insulin, i.e., the cells are not able to take up glucose from the blood stream. This results in rise of glucose level in the blood stream which further causes the increased production of insulin to counteract the glucose in the blood stream. The increased production of insulin ends up in increasing the blood insulin level. This leads to an increased production and heightened activity of hormones such as testosterone and decrease in sex-hormone binding globule (SHBG). Glucose disposal by the action of insulin differs at least six fold in actually healthy individuals, and about one-third of the population that is most sturdy to this activity of insulin is at increased risk to develop a number of adverse clinical outcomes like type 2 diabetes and metabolic syndrome.

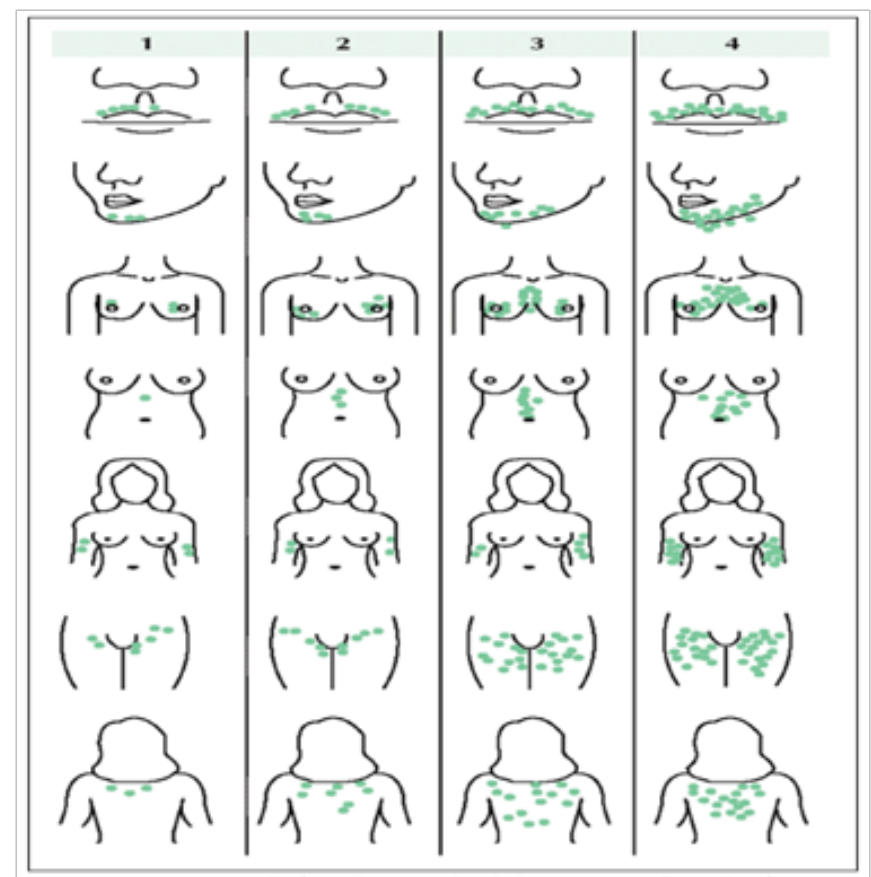

Figure I Hirsutism may be graded from I (minimal hirsutism) to 4 (maximum hirsutism).

\section{Dyslipidemia}

Dyslipidemia is a widespread metabolic abnormality in women with PCOS. ${ }^{8}$ It is a state of abnormal amount of lipids (e.g. cholesterol and/or fat) in blood. It may be characterized by increase in the amount of total cholesterol, triglyceride, low-density lipoprotein (LDL) cholesterol and depletion in the blood concentration of highdensity lipoprotein (HDL) cholesterol. Plaque can build up in the arteries when LDL cholesterol levels are high. With duration of time, this plaque narrows the arteries due to deposition of plaque causing atherosclerosis. This condition can cause heart attacks, heart diseases, peripheral artery disease (reduced flow of blood in the limbs), or stroke. High levels of triglycerides and low levels of HDL too can promote fat accumulation in the arteries. Whereas high levels of HDL cholesterol decreases the accumulation of LDL from the arteries and protects the heart from such diseases. Obesity, hyperandrogenism and insulin resistance coexist in PCOS, and have independent and interactive effects on dyslipidemia. ${ }^{11}$ There is also evidence for heritability of dyslipidemia, so the lipid patterns can be observed not only in women with PCOS but also in their family members. ${ }^{12,13}$

\section{Polycystic ovaries and irregular menstruation}

The ovaries, located on both the sides of uterus of a female, are normally functioned to produce eggs (every month) and hormones, estrogen and progesterone. As shown in Figure 2, during menstrual cycle of normal reproducing women, a follicle is produced in one of the ovaries which carry an immature egg (primary follicle). The primary follicle develops to secondary follicles. The secondary follicle finally turns to a mature follicle carrying a mature egg. The mature follicle then joins the wall of ovary and forms a cyst-like sac filled with egg and necessary fluids. This sac then breaks open, launching the egg into the fallopian tube (ovulation). 


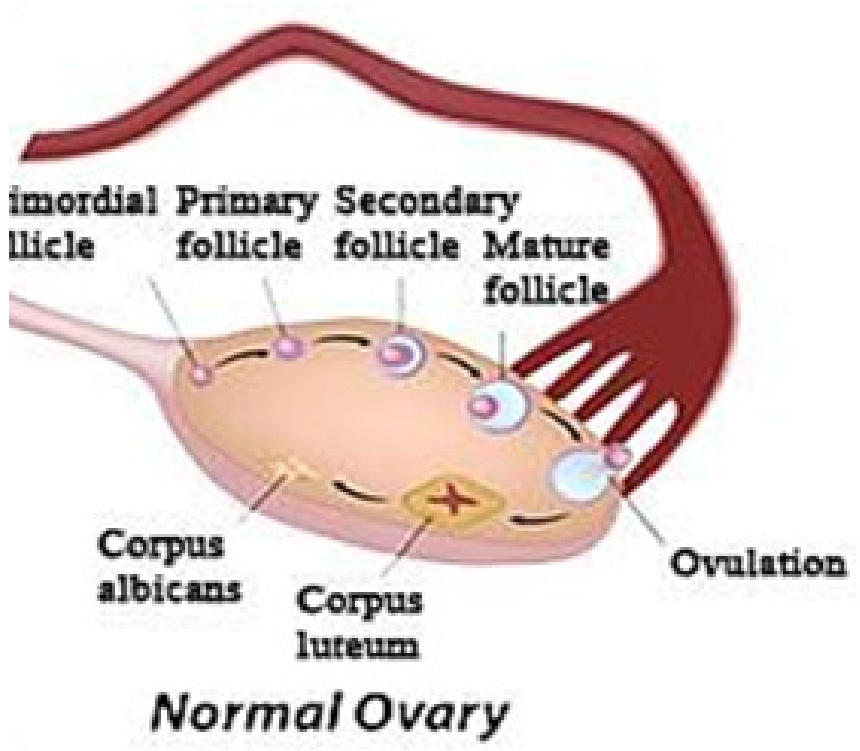

Figure 2 ovulation in ovary of normal reproducing women.

In PCOS condition, follicle development stops at early stage and ovulation is inhibited. The immature follicles turn into cysts and multiple number of cycles leads to the formation of multiple cysts (Figure 3). This may occur due to hormonal imbalance and insulin resistance. The resulting multiple cysts are fluid filled sacs in one or both ovaries and causes the ovary size to enlarge up to twice the size of normal ovary. Polycystic ovaries can be diagnosed by abdominal ultrasound. When the mature egg leaves the ovary, the mature follicle becomes corpus luteum. The corpus luteum prepares the body for the possibility of conception by secreting hormones estrogen and progesterone. When the released egg does not get fertilized, the corpus luteum degenerates leading to a drop in hormones progesterone and estrogen, resulting in menstruation. In case of PCOS, the follicle development stops and there is no release of eggs so the immature follicle becomes cyst and corpus luteum is either not formed (cyst formation) or forms when some immature follicle takes the normal developmental path. Thus, menstruation delays or stops.

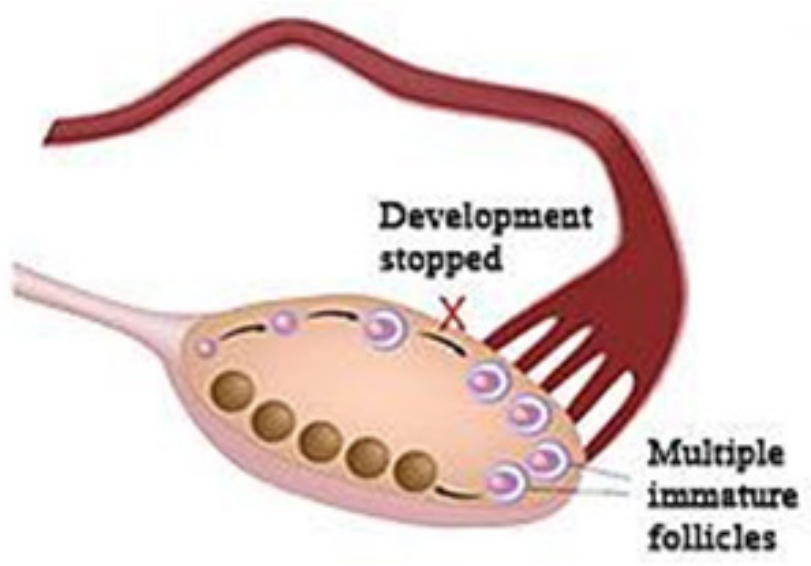

\section{Polycystic Ovary}

Figure 3 The failure in ovulation in polycystic ovary and resulting formation of multiple cysts.

\section{Hypertension}

Blood pressure can be said as the force exerted by the blood on the walls of the blood vessels. The magnitude of this applied force directly based on the cardiac output and the resistance of the blood vessels. Hypertension (HTN or HT), also known as arterial hypertension, high blood pressure, is a medical condition in which blood pressure in the arteries increases dramatically. Increased prevalence of hypertension is a major contributor to the risk for cardiovascular disease and chronic kidney disease. Obesity is a known risk factor for condition of hypertension. One population study demonstrated that blood pressure was more probable to be elated among women who were obese compared to those who were non-obese ( $43.1 \%$ vs. $12.4 \%$, $\mathrm{p}<0.001) .{ }^{8}$ Obesity is one of main diagnostic criteria for women with PCOS. Sustained hypertension over time in these women causes an increased risk of cardiovascular disease as compared to normal reproducing women.

\section{Factors causing PCOS}

\section{Genetic factors}

Genetic factors are widely considered to contribute in causing PCOS. The genes which are considered candidates to contribute to condition of PCOS include androgen receptor(AR), thyroid adenomaassociated (THADA) and DENN domain containing protein 1A (DENND1A), D19S884, CYP 17, luteinizing hormone (LH)/ choriogonadotropin receptor, CYP11A, insulin receptor gene (INSR), sex hormone-binding protein (SHBG), insulin gene variable number of tandem repeats (VNTR), tumor necrosis factor-alpha (TNF-alpha), peroxisome proliferator-activated receptor- $\gamma$ (PPAR- $\gamma$ ), calpain-10, and obesity-associated gene (FTO) ${ }^{14}$ There brief working is as followed:

\section{Follistatin}

Follistatin (FST) is a protein which works for activin-binding that triggers activin in vitro. This ubiquitous protein is present in the highest concentration in ovarian tissue but its role in PCOS is not clear. ${ }^{14}$ For example, circulating FST is increased in PCOS, but its mRNA expression is not there in granulosa cells (GCs). ${ }^{14}$ Attempts to associate endometrial tissue methylation patterns with the promoter region of the FST gene in PCOS were ambiguous. ${ }^{14}$

\section{PPAR- $\gamma$}

PAR- $\gamma$ is included in reproduction and metabolism through its isoforms. The PPAR- $\gamma 1$ isoform regulates ovarian function. PPAR- $\gamma$ transcription is regulated by co-repressors, which are histone deacetylase 3 (HDAC3) and nuclear co-repressor 1 (NCOR1), and co-activators. Studies in PCOS rat models explained that NCOR1 hypo and PPAR- $\gamma 1$ hypermethylated promoters were associated with decreased PPAR- $\gamma 1$ and increased transcription of gene NCOR1 during ovarian maturation and development. ${ }^{14}$ Ovarian dysfunction induced by too much androgen exposure may be influenced by considerably altered methylation of PPAR- $\gamma 1$ and NCOR1 genes and altered HDAC3 acetylation. ${ }^{14}$

\section{CAG Androgen receptor}

The androgen receptor (AR) gene is located at q11-q12 of the $\mathrm{X}$ chromosome and possesses eight exons. Exon 1 contains a polymorphic sequence of CAG repeat which encodes polyglutamine stretches of AR transactivation domain. ${ }^{15,16}$ Evidence implies that CAG number is inversely linked to the transcriptional activity of $\mathrm{AR},{ }^{17,18}$ thus 
greater number of CAG repeats results in decreased testicular atrophy and sperm production. Studies investigated the relationship between PCOS and DNA methylation paid attention on the CAG repeat sequence in Androgenic receptor (AR) gene's exon 1. As AR gene is located on the $\mathrm{X}$ chromosome, any epigenetic modification resulting in activation of the short or the long allele might lead to AR activity that causes heterogeneity, i.e., androgenic features. Differences in localization and DNA methylation pattern of CAG repeat sequence in the AR gene might affect signal transduction and result in development of PCOS [14].

\section{LMNA}

Using Mass ARRAY DNA methylation, the relation between insulin resistance and LMNA gene methylation was examined in PCOS. Major differences were observed in 12/20 cytosine-phosphate-guanine $(\mathrm{CpG})$ sites. Association of hypermethylation with insulin resistance was noticed in PCOS. This gene may play an essential role in the regulation of PCOS-associated insulin resistance. ${ }^{14,18,19}$

\section{LHCGR}

A genome-wide association study was used to study the relationship of PCOS with LH/choriogonadotropin receptor (LHCGR). Wang et al., ${ }^{19}$ considered 192 women with PCOS and sequenced their exons along with flanking regions of LHCGR and reported no somatic mutations. 85 PCOS individuals versus 88 controls were used to measure the methylation of six $\mathrm{CpG}$ sites in the LHCGR promoter region was by pyrosequencing their peripheral blood. This approach identified two hypomethylated sites at -111 and $-174 \mathrm{CpG} .{ }^{14}$ Methylation was additionally examined using GCs. Eight hypomethylated CPG sites $(-174,-61,-148,-43,+10,-8,+17$, and +20$)$ were seen resulting in heightened LHCGR transcription. Hypomethylation in diverse tissues and equivalent expression supports its role in PCOS..$^{14,19}$

\section{EPXHI gene}

A study was done examining three genes concerning steroid synthesis and metabolism. ${ }^{20}$ EPHX1 gene in humans encodes Epoxide hydrolase 1 enzyme which converts reactive epoxides to transdihydrodiols. This protein also plays a key role in the female reproductive system, ${ }^{21-23}$ and two exon single-nucleotide polymorphisms in this gene appear associated with PCOS..$^{14,24}$ Decreased methylation of the EPHX1 promoter region can activate its expression in PCOS. Moreover, it could suppress estradiol (E2) production from testosterone and further increase PCOS risk. ${ }^{14}$

\section{Other genes}

With genome-wide methylated DNA immunoprecipitation technique, Shen et al., ${ }^{25}$ found out that 79 genes were differentially methylated in insulin-resistant as related to non insulin-resistant PCOS. 40 genes were also observed to have different methylation patterns in PCOS as compared to controls. The proceeding examination by pathway enrichment analysis and gene ontology (GO) showed that immune response classes were differentially methylated. It was observed that in these patients the cancer pathway genes were differentially methylated as well. Wang et al.,26 using DNA methylation profiling along with transcriptome analysis, found out that there were about 650 differential transcripts and minimum 3\% differentially methylated sites and in PCOS ovaries as compared to normal ovaries. The fibrillin 1 (FBN1), which was hypermethylated and upregulated, and, chemokine (C-C motif) ligand 2 (CCL2), which was hypermethylated and downregulated, have been correlated with PCOS. ${ }^{14}$

\section{Epigenetic regulation of traf2- and Nck-interacting kinase (TNIK)}

Da Li et al., ${ }^{27}$ did a study with tissues of ovaries from PCOS patients and normal ovaries; patients with PCOS reported that the TNIK transcript was up regulated in PCOS ovarian tissues versus normal ovarian tissues and methylation of $\operatorname{cg} 10180092$ site is a key regulatory element for TNIK transcription. The molecular mechanism can include hypermethylated cg10180092 site-mediated loss of the repressive histone marker $\mathrm{H} 3 \mathrm{~K} 9$ me enrichment, which activates the transcription of TNIK. ${ }^{27}$ TNIK is an activator of Wnt signaling and Wnt signaling pathway (also its key components) have critical roles in regulation of many cellular functions. It has been recently highlighted that Wnt signaling have a role in metabolic homeostasis, and the misregulation of metabolic homeostasis leads to conditions often involved in PCOS pathophysiology such as obesity, insulin resistance, and inflammation and lipoprotein metabolism. PCOS is characterized by proliferative disorders like follicle growth arrest, endometrial proliferation, cumulus cells and abnormal granulosa cells; TNIK is a regulator of proliferation and siRNA-mediated silencing of TNIK causes inhibition of cell proliferation and induction of cell death. Therefore, functional variability of TNIK might be involved in PCOS-associated proliferative disorders.

\section{Genes involved in androgen and insulin resistance}

As biochemical phenotype is known for patient with polycystic ovaries, Stephen Franks et al. ${ }^{28}$ focused on genes coding for steroidogenic enzymes in the androgen biosynthetic pathway and those involved in the action as well as secretion of insulin ${ }^{27}$ they are as followed:

Genes coding for steroidogenic enzymes: CYP17 gene codes for 7-hydroxylase/17, 20-lyase which is a rate limiting step in the biosynthesis of androgen. On the basis of procedures of amplification and screening, Stephen Franks et al., ${ }^{28}$ were able to exclude CYP17 as a major causative gene but in none of their studies, any relationship was found between the CYP17 variant and serum androgen levels.

PCO theca cells produce an excess of both androgens and progesterone. This made Stephen Franks et al., ${ }^{28}$ to study CYP11a. On the basis of amplification and genotype analysis of PCOS patient's theca cells to show that variation at the CYP11agene was related to both serum testosterone concentrations and PCOS.

There have been reports of hyperandrogenism striking in rare patients with aromatase deficiency (Harada et al., 1992; Ito et al., 1993). So possible role of the gene CYP19 (encoding P450 aromatase) was examined. All the studies pointed to abnormal regulation of aromatase in women suffering from hyperandrogenism. Stephen Franks et al., ${ }^{28}$ therefore presented a case control study as well as linkage analysis. The results disclosed no relation of alleles of CYP19 with PCO and no evidence for excess allele sharing. ${ }^{28}$

Genes involved in secretion and action of insulin: The validation of impaired sensitivity to insulin action in vivo and in vitro naturally led to the hypothesis that insulin receptor's genetic abnormalities and post-receptor signaling were involved in the pathogenesis of familial PCOS. ${ }^{28}$ By various case studies and resulting polymorphic analysis, the authors reported that mutations of insulin receptor gene were infrequent in women with PCOS.

Insulin secretion abnormalities have been reported in recent studies of women with PCOS. Therefore, Stephen Franks et al., ${ }^{28}$ inves- 
tigated the role of the insulin gene in the causes of PCOS. ${ }^{28}$ It was found that Class III alleles were related with PCOS in all the cases. An additional finding was that insulin VNTR class III alleles were strongly related with anovulatory PCOS. This is in context with the observation that hyperinsulinemia is a noticeable feature in women with polycystic ovaries who have an ovulatory menses (or amenorrhea) than in alike hyperandrogenaemic subjects with regular menses (Dunaif et al., 1987; Robinson et al., 1993).

\section{Present treatments}

\section{Drug Metformin and oral contraceptive pills (OCPs)}

Metformin is a commonly used drug in the maintenance of type 2 diabetes and has also been used in the treatment of metabolic derangements in PCOS. The clinical trials reviewed in the study conducted by Young-Mo Yang et al., ${ }^{29}$ showed that metformin or OCPs have the potential to control the metabolic symptoms in women with PCOS. They are last efficacious and safe patient-convenient medications for the treatment of PCOS. Metformin helps to decrease in BMI of 1 to $2 \mathrm{~kg}$ per $\mathrm{m}^{2}$ of the patient. ${ }^{4}$ It is necessary to monitor PCOS women on these medications since conditions such as lactic acidosis or weight gain can occur by the long-term use of them. ${ }^{29}$

\section{Glucocorticoids}

Glucocorticoids (dexamethasone or prednisone) suppress ACTHdependent adrenal androgen synthesis. It is used for treating women with adrenal hirsutism (congenital adrenal hyperplasia (CAH) or idiopathic adrenal hyperandrogenism). Some patients gain weight and bear Cushing syndrome, even with a small dose of 0.5-1mg. Further investigations may base that lower doses $(\sim 0.25 \mathrm{mg})$ can be working without adverse effects.

\section{Spironolactone}

Spironolactone $(50-200 \mathrm{mg}$ daily) blocks androgen receptors. Spironolactone also reduces the testosterone production, becoming a solution for hirsutism as well. Spironolactone is primarily useful in a patient with hypertension.

\section{Finasteride}

Finasteride is a 5-alpha-reductase inhibitor authorized for the treatment of benign prostatic hyperplasia. No untimely effects have been seen in women, and the potency is similar to that of spironolactone. This drug is only used in postmenopausal women as usage of this drug has risk of ambiguous genitalia in fetuses in first trimester.

\section{Flutamide}

Flutamide, one of the newer therapies, is a potent nonsteroidal selective antiandrogen without progestational, estrogenic, corticoid, or antigonadotropin activity. It is adequate as therapy for hirsutism (and also acne); however, flutamide is uneconomical and has side effects.

\section{Cyproterone}

Cyproterone acetate has been effective in the treatment of hirsutism. When added to ethinyl estradiol, it is as compelling as flutamide in the treatment of hirsutism.

\section{Exercising}

Currently, a healthy diet routine and physical exercise is advised as first-degree therapy for PCOS patient, importantly for the obese or overweight PCOS women. Joceline C.F. Sá et al., ${ }^{30}$ studied that aerobic exercise has some benefits on the cardio metabolic risk factors in women with PCOS, including betterment in insulin resistance, visceral obesity blood pressure, cardio respiratory fitness, dyslipidemia and inflammatory pattern. Additionally, aerobic exercise can help in betterment of cardiac autonomic system function in the women with PCOS.

\section{Acupuncture}

Study by Julia Johansson et al., ${ }^{31}$ is about acupuncture being a potential treatment alternative for reproductive and endocrine disorders in women with PCOS. Several animal and clinical experimental studies recorded that acupuncture is beneficial for ovulatory dysfunction in PCOS. However, the probability of changes in sex steroids and ovulation is tough to determine. The decrease of androgen levels by normalization of intrinsic ovarian abnormalities may relax the exaggerated follicular growth, restore the follicle maturation processes, and hence lead to ovulation. ${ }^{31}$

\section{Laparoscopic ovarian drilling}

When ovulation is not induced with any therapy, ovulation induction can be done by other techniques as an infertility treatment. Laparoscopic ovarian drilling (LOD) is a prosperous technique in generating a pregnancy in likely of $84 \%$ of PCOS patients and helps improve ovarian androgen production and insulin resistance, also increases SHBG levels. ${ }^{32,33}$

\section{Future perspective}

Despite of ages of research and experiments conducted, limited work has been done that covers the whole PCOS spectrum and thus the data about polycystic syndrome is not very convincing as there are no prominent causes and thus no medicinal cure. One of the most challenging sides of PCOS is its complexity of characteristics and vague diagnostics for future a lot of research is required in genetics field and pathophysiology of PCOS to determine its cause, prevent its risk factors and resultantly generate its successful cure and treatment.

\section{Acknowledgments}

None.

\section{Conflicts of interest}

The author declares there are no conflicts of interest.

\section{References}

1. Sam S. Obesity and polycystic ovary syndrome. Obes manag. 2007;3(2):69-73.

2. Kollmann M, Martins WP, Raine-Fenning N. Terms and thresholds for the ultrasound evaluation of the ovaries in women with hyperandrogenic anovulation. Hum Reprod Update. 2014;20(3):463-464.

3. Kim JJ, Choi YM. Dyslipidemia in women with polycystic ovary syndrome. Obstet Gynecol Sci. 2013;56(3):137-142.

4. Radosh L. Drug treatments for polycystic ovary syndrome. American family physician. 2009;79(8):671-676.

5. Teede H, Deeks A, Moran L. Polycystic ovary syndrome: a complex conditions with psychological, reproductive and metabolic manifestations that impacts on health across the lifespan. BMC med. 2010;8:41.

6. Diamanti-Kandarakis E, Kandarakis H, Legro RS. The role of genes and environment in the etiology of PCOS. Endocrine. 2006;30(1):19-26. 
7. Dabhade Suhas, Suresh Dabhade, Panchshila Dabhade. Role of glycemic control on lipids profile in Diabetic Sudanese patients. Journal of Science Medicine. 2016;6(4):208-212.

8. Reaven GM. The insulin resistance syndrome: definition and dietary approaches to treatment. Annu Rev Nutr. 2005;25:391-406.

9. Baptiste CG, Battista MC, Trottier A, et al. Insulin and hyperandrogenism in women with polycystic ovary syndrome. J Steroid Biochem Mol Biol. 2010;122(1):42-52.

10. Joshi Mehak. Polycystic Ovary Syndrome (PCOS). International Journal of Scientific \& Engineering Research. 2014;5(12):514-521.

11. Diamanti-Kandarakis E, Papavassiliou AG, Kandarakis SA, et al. Pathophysiology and types of dyslipidemia in PCOS. Trends in Endocrinol Metab. 2007;18(7):280-285.

12. Sam S, Legro RS, Bentley-Lewis R, et al. Dyslipidemia and metabolic syndrome in the sisters of women with polycystic ovary syndrome. $J$ Clin Endocrinol Metab. 2005;90(8):4797-4802.

13. Hung JH, Hu LY, Tsai SJ, et al. Risk of psychiatric disorders following polycystic ovary syndrome: a nationwide population-based cohort study. PloS One. 2014;9(5):e97041.

14. Ilie IR, Georgescu CE. Chapter Two-Polycystic Ovary Syndrome-Epigenetic Mechanisms and Aberrant MicroRNA. Advances in clinical chemistry. 2015;71:25-45.

15. Dasgupta S, Sirisha PVS, Neelaveni K, et al. Androgen receptor CAG repeat polymorphism and epigenetic influence among the south Indian women with Polycystic Ovary Syndrome. PLoS One. 2010;5(8):e12401.

16. Tirabassi G, delli Muti N, Corona G, et al. Androgen receptor gene CAG repeat polymorphism regulates the metabolic effects of testosterone replacement therapy in male postsurgical hypogonadotropic hypogonadism. International journal of endocrinology. 2013(2013):1-7.

17. Chamberlain NL, Driver ED, Miesfeld RL. The length and location of CAG trinucleotide repeats in the androgen receptor N-terminal domain affect transactivation function. Nucleic acids Res. 1994;22(15):31813186.

18. Ting W, Yanyan Q, Jian H, et al. The relationship between insulin resistance and $\mathrm{CpG}$ island methylation of LMNA gene in polycystic ovary syndrome. Cell Biochem Biophys. 2013;67(3):1041-1047.

19. Wang P, Zhao H, Li T, et al. Hypomethylation of the LH/choriogonadotropin receptor promoter region is a potential mechanism underlying susceptibility to polycystic ovary syndrome. Endocrinology. 2014;155(4):1445-1452.

20. Hartsfield JK, Sutcliffe MJ, Everett ET, et al. Assignment1 of microsomal epoxide hydrolase (EPHX1) to human chromosome $1 \mathrm{q} 42.1$ by in situ hybridization. Cytogenet Cell Genet. 1998;83(1-2):44-45.
21. Wang X, Wang M, Niu T, et al. Microsomal epoxide hydrolase polymorphism and risk of spontaneous abortion. Epidemiology. 1998;(5):540 544.

22. Lancaster JM, Brownlee HA, Bell DA, et al. Microsomal epoxide hydrolase polymorphism as a risk factor for ovarian cancer. Mol Carcinog. 1996;17(3):160-162.

23. Zusterzeel PL, Peters WH, Visser W, et al. A polymorphism in the gene for microsomal epoxide hydrolase is associated with pre-eclampsia. $J$ Med Genet. 2001;38(4):234-237.

24. Korhonen S, Romppanen EL, Hiltunen M, et al. Two exonic single nucleotide polymorphisms in the microsomal epoxide hydrolase gene are associated with polycystic ovary syndrome. Fertil Steril. 2003;79(6):1353-1357.

25. Shen HR, Qiu LH, Zhang ZQ, et al. Genome--wide methylated DNA immunoprecipitation analysis of patients with polycystic ovary syndrome. PLoS One. 2013;8(5):e64801.

26. Wang XX, Wei JZ, Jiao J, et al. Genome-wide DNA methylation and gene expression patterns provide insight into polycystic ovary syndrome development. Oncotarget. 2014;5(16):6603-6610.

27. Li D, Jiao J, Zhou YM, et al. Epigenetic regulation of traf2-and Nck-interacting kinase (TNIK) in polycystic ovary syndrome. Am J Transl Res. 2015;7(6):1152-1160.

28. Stephen Franks, Neda Gharani, Dawn Waterworth, et al. The genetic basis of polycystic ovary syndrome. Human Reproduction. 1997;12(12):2641-2648.

29. Yang YM, Choi EJ. Efficacy and safety of metformin or oral contraceptives, or both in polycystic ovary syndrome. Ther Clin Risk Manag. 2015;11:1345-1353.

30. Sá JC, Costa EC, da Silva E, et al. Aerobic exercise improves cardiac autonomic modulation in women with polycystic ovary syndrome. Int $J$ Cardiol. 2016;202:356-361.

31. Johansson J, Stener-Victorin E. Polycystic Ovary Syndrome: Effect and Mechanisms of Acupuncture for Ovulation Induction. Evid Based Complement Alternat Med. 2013:762615.

32. Ferlin A, Bartoloni L, Rizzo G, et al. Androgen receptor gene CAG and GGC repeat lengths in idiopathic male infertility. Mol Hum Reprod. 2004;10(6):417-421.

33. Puurunen J, Piltonen T, Morin-Papunen L, et al. Unfavorable hormonal, metabolic, and inflammatory alterations persist after menopause in women with PCOS. J Clin Endocrinol Metab. 2011;96(6):1827-1834. 